OPEN ACCESS

Edited by:

Justin Zhan,

University of Arkansas, United States

Reviewed by:

Indu Khatri,

Leiden University Medical

Center, Netherlands

Yaojiang Huang,

Minzu University of China, China

*Correspondence:

Kuppan Gokulan

Kuppan.gokulan@fda.hhs.gov

Specialty section

This article was submitted to Medicine and Public Health,

a section of the journal

Frontiers in Artificial Intelligence

Received: 18 November 2020 Accepted: 01 March 2021

Published: 25 March 2021

Citation:

Khare S, Azevedo M, Parajuli P and Gokulan K (2021) Conformational

Changes of the Receptor Binding

Domain of SARS-CoV-2 Spike Protein and Prediction of a B-Cell Antigenic

Epitope Using Structural Data.

Front. Artif. Intell. 4:630955.

doi: 10.3389/frai.2021.630955

\section{Conformational Changes of the Receptor Binding Domain of SARS-CoV-2 Spike Protein and Prediction of a B-Cell Antigenic Epitope Using Structural Data}

\author{
Sangeeta Khare, Marli Azevedo, Pravin Parajuli and Kuppan Gokulan* \\ Division of Microbiology, National Center for Toxicological Research, United States-Food and Drug Administration, Jefferson, \\ AR, United States
}

COVID-19, the illness caused by the SARS-CoV-2 virus, is now a worldwide pandemic with mortality in hundreds of thousands as infections continue to increase. Containing the spread of this viral infection and decreasing the mortality rate is a major challenge. Identifying appropriate antigenic epitopes from the viral proteins is a very important task for vaccine production and the development of diagnostic kits and antibody therapy. A novel antigenic epitope would be specific to the SARS-CoV-2 virus and can distinguish infections caused by common cold viruses. In this study two approaches are employed to identify both continuous and conformational B-cell antigenic epitopes. To achieve this goal, we modeled a complete structure of the receptor binding domain (RBD) of the spike protein using recently deposited coordinates (6vxx, 6vsb, and 6w41) in the protein data bank. In addition, we also modeled the RBD-ACE2 receptor complex for SARS-CoV-2 using the SARS-CoV RBD-ACE2 complex (3DOJ) as a reference model. Finally, structure based predicted antigenic epitopes were compared to the ACE2 binding region of RBD of SARS-CoV-2. The identified conformational epitopes show overlaps with the ACE2-receptor binding region of the RBD of SARS-CoV-2. Strategies defined in the current study identified novel antigenic epitope that is specific to the SARS-CoV-2 virus. Integrating such approach in the diagnosis can distinguish infections caused by common cold viruses from SARS-CoV-2 virus.

Keywords: spike protein, SARS-CoV-2, receptor binding domian, conformational epitope, viral infection

\section{INTRODUCTION}

The first incidence of COVID-19 was reported in China in late December 2019. The COVID-19 infection caused symptoms of severe acute respiratory syndrome, severe respiratory illness, fever, and pneumonia (Huang et al., 2020; WHO, 2020; Zhou et al., 2020; Zhu et al., 2020). The causative agent for this disease is designated as SARS coronavirus-2 (SARS-CoV-2). The ongoing SARSCoV-2 illness was declared a global pandemic with mortality in the hundreds of thousands and infections continuously increasing every day. The rate of mortality is much higher in individuals 
who have preexisting health issues that include cancer, hypertension, diabetes, coronary heart disease, liver disease, and pre-existing digestive diseases. The genetic material of SARS$\mathrm{CoV}-2$ is a single-strand positive sense RNA, and the so-called spike protein protrudes from the envelope layer. The genome analysis reveals that SARS-CoV-2 belongs to the same subgenus of SARS-CoV (Sarbecovirus), thus it has been classified as a new member of betacoronavirus genus (Xu et al., 2020). The hosts for coronaviruses are birds and mammals, but they have crossed the species barrier and evolved as a zoonotic pathogen (Anthony et al., 2017; Zhou et al., 2020). Both SARS-CoV and SARS-CoV-2 spread through human to human contact. However, the rate of viral transmission and infectivity of SARS-CoV-2 is far greater compared to SARS-related coronavirus (Chen et al., 2020).

Like SARS-CoV, the spike protein of SARS-CoV-2 also initiates binding with ACE2 receptors on the host cells through its spike protein and it replicates in primary airway epithelial cells, lungs, and the gastrointestinal system (Phan, 2020). Both SARS$\mathrm{CoV}$ and Middle East Respiratory Syndrome-CoV (MERS-CoV) are etiological agents for an acute respiratory syndrome that has caused mortality in the last two decades (Gralinski et al., 2018). In 2003, SARS-CoV caused a mortality rate of $9.6 \%(774 / 8,076)$ (WHO, 2015), whereas in 2013 MERS-CoV infection caused a mortality rate of around $34.4 \%(858 / 2,494)$ (WHO, 2015, 2019). As of April 27, 2020, SARS-CoV-2 caused infection in 3,029,736 individuals and the mortality of 209,244 individuals (7\% CMR). It is evident that the SARS-CoV-2 pathogenesis might be different from that of SARS-CoV and MERS-CoV coronaviruses. Recent pathological studies reveal that SARS-CoV-2 causes alveolar damage, edema, type-II hyperplasia pneumonia, inflammation, and acute respiratory distress syndrome (ARDS) (Xu et al., 2020). However, these viruses use the spike protein to enter host cells. The spike protein is composed of S1 and S2 domains. The S1 domain plays an essential role in the initial binding with host cell receptors, whereas the S2 domain is responsible for the fusion and entry of the virus into host cells (Gui et al., 2017; Tortorici and Veesler, 2019). The structure of the spike protein of SARS-CoV-2 was published by cryo-electron microscopy (cryo-EM) (Walls et al., 2020; Wrapp et al., 2020). The solved structures provide evidence that the spike proteins exist in two conformations [closed (pre-fusion) and open (post-fusion)]. It is evident that the spike protein undergoes conformational changes to interact with the ACE2 receptor. This manuscript focuses mostly on the conformational changes between the open and closed structure of the spike protein of SARS-CoV-2, and in particular to the receptor binding domain (RBD).

The structural information reveals that the S1 domain is primarily composed of $\beta$-strands in the center (Wrapp et al., 2020). Both sides of $\beta$-strands are surrounded by several $\alpha$ helices, and it folds into a V shaped architecture. Particularly, the structural fold shows that the receptor binding domain (RBD) packs against the $\mathrm{N}$-terminal region. These two regions are connected by a hinge loop that allows them to fold against each other. In addition, the RBD is covered with a flexible loop conformation on the top of its globular head. The S2 domain is mostly composed of both $\alpha$-helices and $\beta$-strands and is highly glycosylated. Similar to $\mathrm{S} 1$, the $\beta$-strands in S2 also occupy the center of the core of trimer and are surrounded by $\alpha$-helices on the outside. These two domains are connected by a long flexible loop that provides these domains movement and flexibility. Three monomeric subunits of spike protein assemble and form a functional unit, which is attached to the envelope protein through the transmembrane region (C-terminal region). Upon assembly, the RBD occupies the top of the homotrimer and forms a globular head and protrudes from the viral cell surface. The N-terminal region (1-300 amino acids) of S1 occupies just below the RBD 42 $\AA$ away from the core of the homotrimer. It is important to note that among all coronaviruses, amino acids of the S1 domain are highly variable and few residues are conserved. The N-terminal portion of the S1 domain (residues 1-350) is highly glycosylated, whereas the S2 domain is intermittently glycosylated within the entire region that initiates viral fusion inside the host cells. Unlike the S1 domain, residues at the S2 domain are highly conserved in various coronaviruses.

\section{METHODS}

\section{Homology Modeling of SARS-CoV-2 Structure}

Atomic coordinates for SARS-CoV-2 spike protein are available in the Protein Data Bank (PDB), and 6vsb and 6vxx coordinates were downloaded from the PDB http://www.rcsb.org/pdb. The crystal structure of the RBD of SARS-CoV-2 complexed with monoclonal antibody has been recently deposited (PDB 6w41) (Yuan et al., 2020) in PDB. This antigen-antibody complex structure was solved at a higher resolution with a complete structure of the RBD of SARS-CoV-2. The RBD was extracted from the complex structure (PDB 6w41) for structural superposition on top of the RBD from the $6 \mathrm{svb}$ and $6 \mathrm{vxx}$ coordinates to get a complete structural fold of RBD of SARS$\mathrm{CoV}-2$. Earlier, the crystal structure of a chimeric molecule of the ACE2 receptor complexed with RBD of SARS-CoV (3D0J) was reported ( $\mathrm{Li}, 2008)$. The 3D0J coordinate was downloaded from the $\mathrm{PBD}$ as a reference model. The chimeric molecule of the ACE2 receptor complexed with the RBD of SARS-CoV was used as a reference model to build RBD-ACE2 complex for SARS-CoV-2. The modeled binary complex structure was used for analyzing the interaction between ACE2 with RBD complete structure. Before structural comparison of this complex, these modeled structures were energy minimized in the Insight II suite using Discovery (Accelrys). Win coot, Pymol, chimera, and Insight II suite were used for conducting structural analysis and comparison and generating figures.

\section{Conformational and Linear Antigenic Epitope Prediction}

Genome sequence retrieval: We downloaded SARS genome sequence and protein sequence for BAT, SARS-61-TW. SARSWahun-2, SARS-USA, SARS-ITA, and SARS-Ind from NCBI GenBank. Multiple sequence alignment was performed in CLUSTAL-OMEGA online software to identify the sequence homology, \% of conserved and non-conserved residues (http:// www.ebi.ac.uk/support/sequence-alignment). Preceding section 

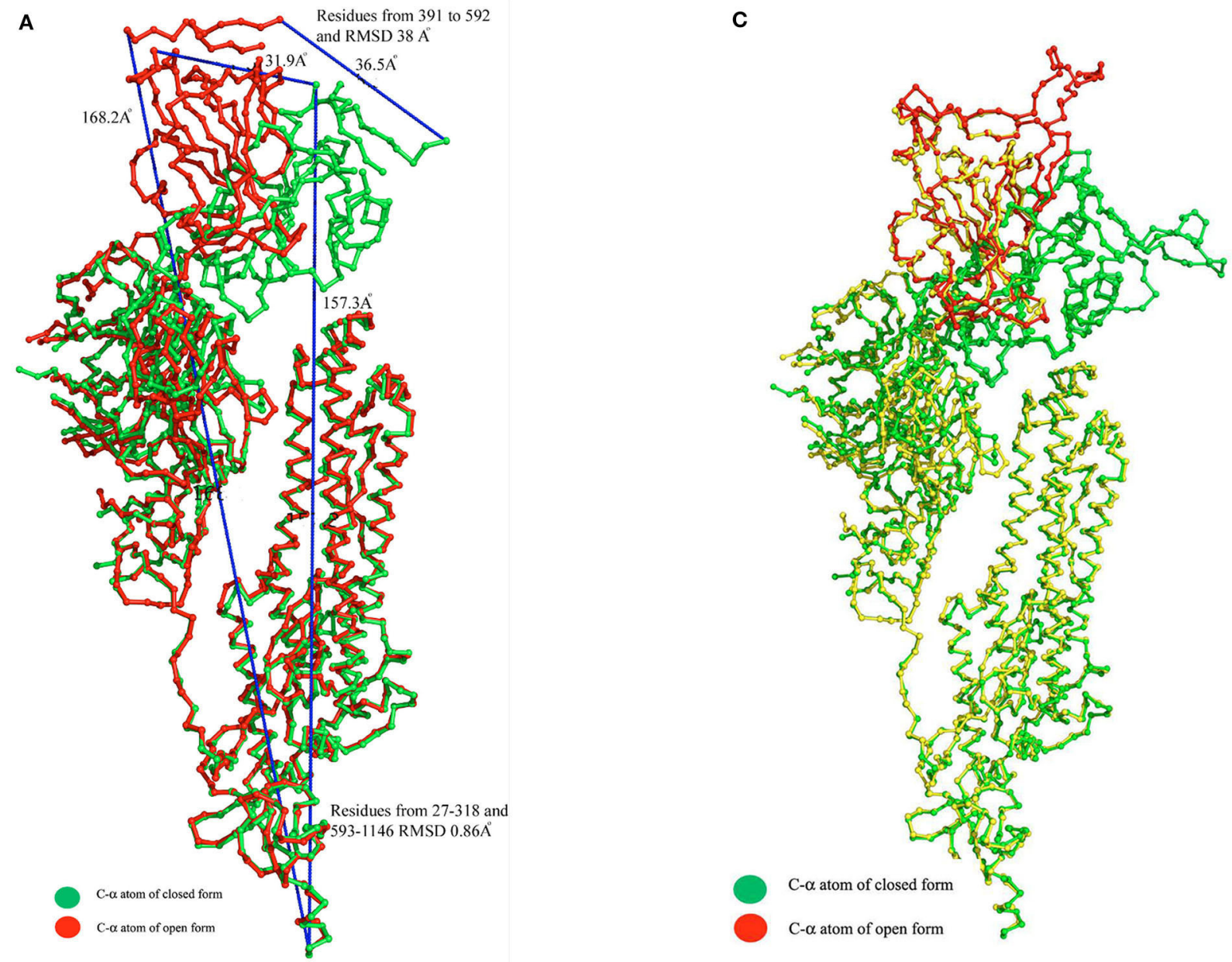

B

D
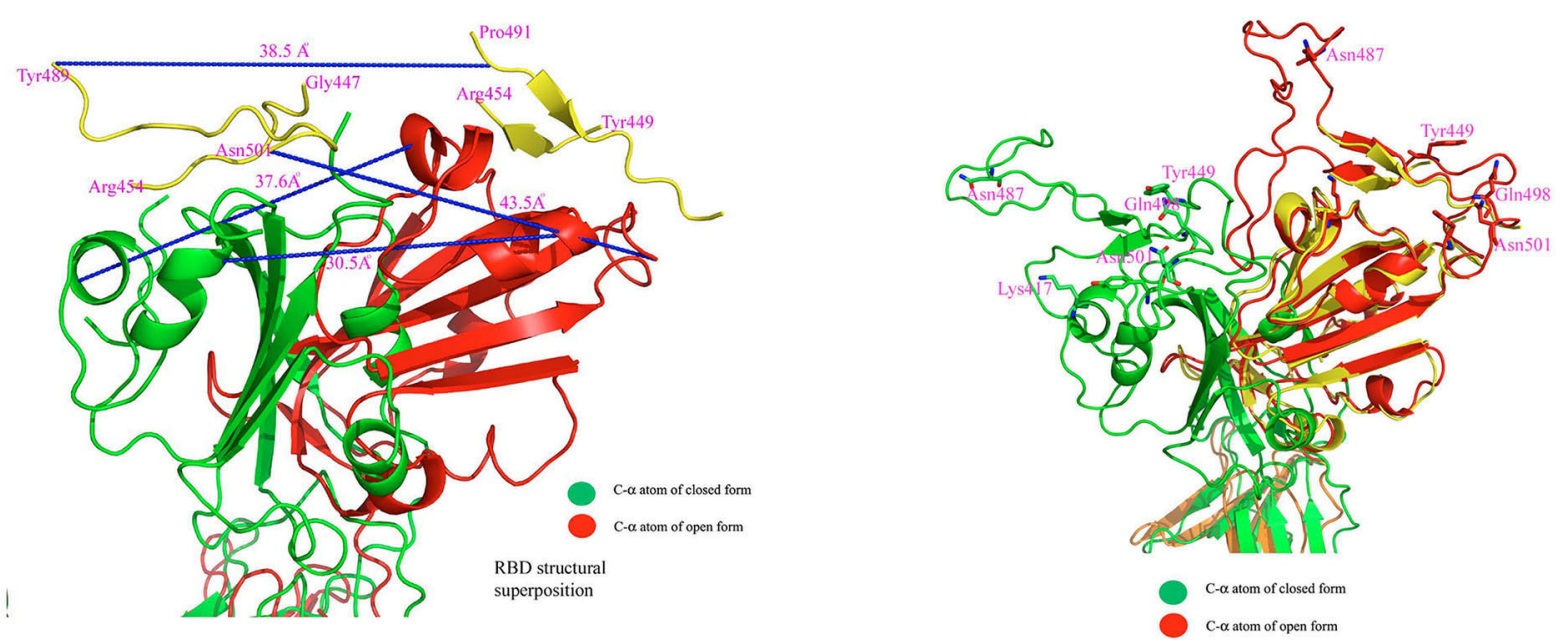

FIGURE 1 | (A) C- $\alpha$ atom superposition of 6vsb with 6vxx coordinates. This alignment shows C- $\alpha$ atoms from residues $27-318$ and $563-1146$ aligns on top of each other with root mean square deviation (rmsd) $0.86 \AA$. (B) Magnified cartoon diagram that shows only RBD of both structures with superposition and RMSD between 
FIGURE 1 | these two structures is $\sim 38 \AA$. The globular head shows the missing loops region in both structures and part of the region is shown in yellow color. Color code: Green color-closed form and red color-open form. (C) Structural superposition of the RBD of 6w41 coordinates on top of RBD of 6vxx and 6vsb. (D) Cartoon diagram shows the superposition of the RBD of 6w41 coordinates on top of both structural forms. Color code: Green color-closed form, red- (complete structure of RBD from pdb 6w41), and yellow (RBD lack loops) the open form. The distance between these two structures are also shown in dotted lines.

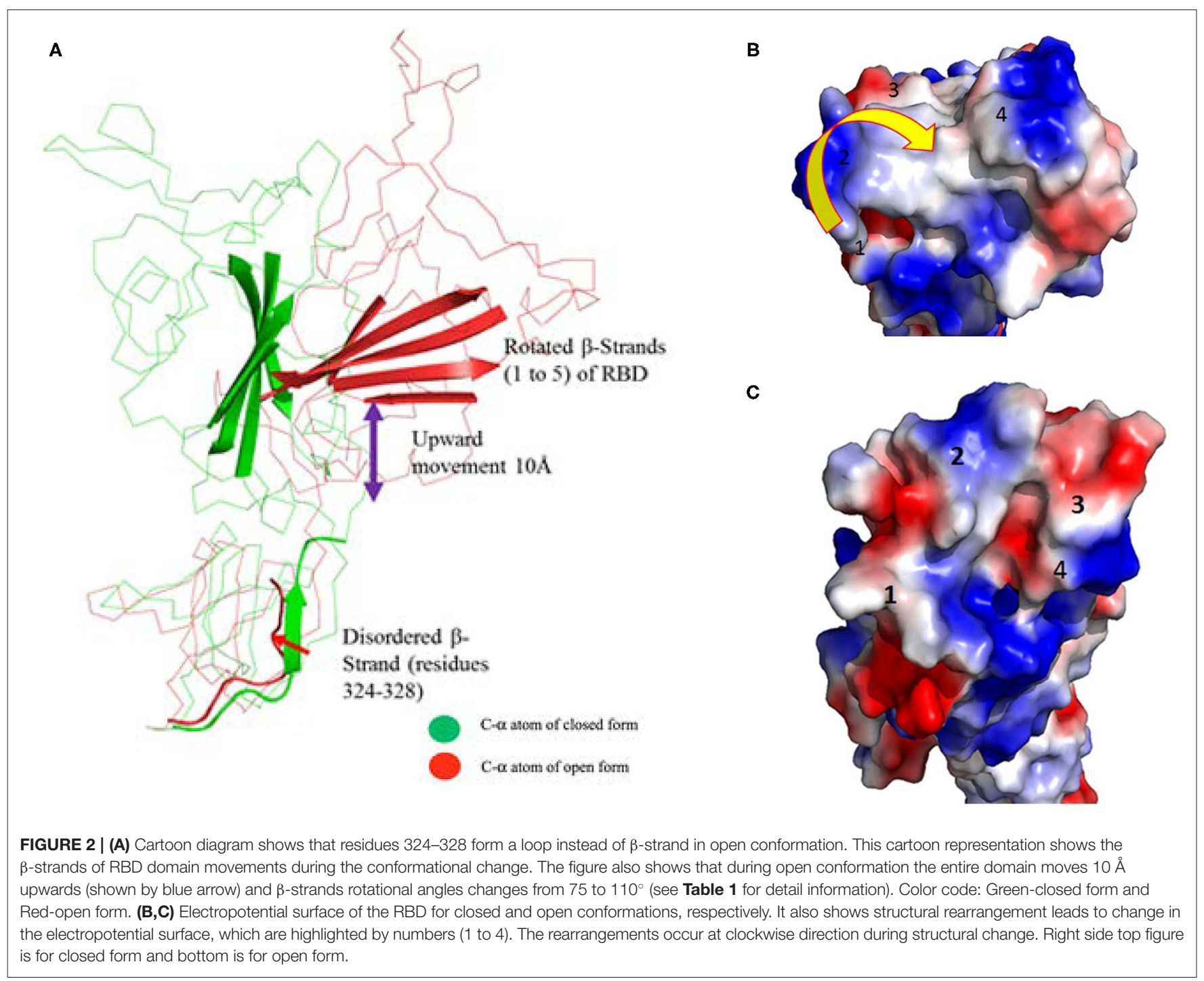

describes the complete homology modeling and building of SARS-CoV2 spike protein structure using various PDB coordinates. The modeled spike protein was energy minimized and inspected by structural superposition with Cryo-EM structure of SARS-CoV2 spike protein. To identify structure based conformational antigenic epitope prediction, we used two different bioinformatic tools based on the surface accessibility and propensity (Haste Andersen et al., 2006; Ponomarenko et al., 2008). To identify linear B-cell antigenic or continuous B-cell epitopes online bioinformatic tools (http://tools.iedb.org/bcell) was used. The antigenic epitopes were predicted using several methods that utilize various parameter. The protein sequence was submitted to predict the antigenic epitope based on the criteria that include beta-turn predictions, surface accessibility, flexibility, and hydrophilic scale (Chou and Fasman, 1978; Hopp and Woods, 1983; Emini et al., 1985; Karplus and Schulz, 1985; Parker et al., 1986; Kolaskar and Tongaonkar, 1990; Lins et al., 2003; Jespersen et al., 2017). For example, Emini predicts the protein sequence based on surface accessibility, whereas BepiPred-2.0 server predicts B-cell epitope from the protein sequence using Random Forest algorithm and trained antigenic epitope from the crystal structure. Both conformational and linear antigenic epitopes were further analyzed for allergenicity using Allercatpro (https://allercatpro.bii.a-star.edu.sg/). 


\section{RESULTS AND DISCUSSION}

\section{Structural Analysis of Open and Closed Conformation}

Recently the structure of SARS-CoV2 spike protein has been solved by two groups using cryo-EM (Walls et al., 2020; Wrapp et al., 2020). The McClellan laboratory has shown that all subunits of trimeric complex are either in closed conformation (pre-fusion stage) or open conformation (post fusion) of the functional unit (Wrapp et al., 2020). Walls et al. (2020) have shown that one subunit is in open form and the other two subunits are in closed form. Both structures reveal that a homotrimer is the functional unit for binding with ACE2 receptors. While comparing the structural superposition of these two structures, C- $\alpha$ atoms from residues $27-318$ and 593-1,273 align very well between the open and closed conformation with root mean square deviation (rmsd) $0.86 \AA$. In contrast, C- $\alpha$ atoms from residues 319-592 did not align well and show major structural differences between these two conformations with a rmsd of $\sim 38 \AA$ (Figure 1A). Both cryo-EM structures provided a wealth of information but lack several connecting loop regions in the S1 domain. Particularly, the missing loop regions are located near the globular head, which is essential for ACE2 receptor binding (Figure 1B). In order to get complete structural information of the RBD of SARS$\mathrm{CoV}-2$, the crystal structure of the SARS-CoV-2 RBD domain of $6 \mathrm{w} 41$ coordinate was superimposed on top of both the closed and open form of SARS-CoV-2 spike protein structures individually to get the complete structural information of RBD (Figure 1C). Obtaining complete structural information of the RBD will provide a better understanding of the structural rearrangement between these two forms and it can also provide a clear picture of the interaction with the ACE2 receptor molecule (Figure 1D).

The superimposed model had a complete structure of the RBD of SARS-CoV-2, so it was used for further structural analysis. The RBD is connected by a long loop (319-337) containing a short $\beta$-strand (residues from 324-338) in the middle of the closed form (Figure 2A). In contrast, no ordered $\beta$-strand is found in the open form and part of the region is disordered. The alignment also reveals a $2.6 \AA$ rmsd between them at amino acid position 319 . The structural movement is gradually increased until the globular head as shown in Figures 1A,B. The rmsd at the globular head is $\sim 38 \AA$ between the open and closed conformation. In addition, in open conformation the RBD moved up and outward from the core structure in comparison with closed conformation. The RBD is composed of five anti-parallel $\beta$-strands (named as $\beta 1$ to $\beta 5$ ) (Table 1) in the middle and surrounded by several $\alpha$-helices at the outside. The structural movement of each individual $\beta$-strand of RBD is further compared and analyzed for upward movement and rotational angle as well (Figure $\mathbf{2 A}$ ). The table shows the structural movement of individual $\beta$-strands of the RBD between these two forms. We also analyzed the electropotential surface between the closed and open conformation of the RBD of SARS$\mathrm{CoV}-2$. The structural rearrangement leads to change in the electropotential surface on the globular head. Particularly, the globular head region rotates in a clockwise direction as shown by numbers (labeled as 1 to 4 ) in Figures 2 B,C.
TABLE 1 | Conformational changes and rotational angles of $\beta$-stands of RBD between open and closed conformation.

\begin{tabular}{lccccc}
\hline$\beta$-strand & $\begin{array}{c}\text { Residues } \\
\text { closed } \\
\text { form }\end{array}$ & $\begin{array}{c}\text { Residues } \\
\text { open form }\end{array}$ & $\begin{array}{c}\text { Rotational } \\
\text { angle }\end{array}$ & $\begin{array}{c}\text { Upward } \\
\text { movement } \\
\AA\end{array}$ & $\begin{array}{c}\text { Distance } \\
\text { from } \\
\text { starting } \\
\text { point to } \\
\text { end } \AA\end{array}$ \\
\hline$\beta 1$ & $354-358$ & $354-358$ & 75 & 3.8 & $6.9 \& 15.6$ \\
$\beta 2$ & $375-379$ & $375-380$ & 109 & 9.7 & 11.1 \\
$\beta 3$ & $393-403$ & $391-403$ & 90 & 3.8 & $3.6 \& 31.3$ \\
$\beta 4$ & $431-437$ & $431-437$ & 102 & 9.9 & 10.2 \\
$\beta 5$ & $507-516$ & $507-516$ & 107 & 12.9 & 12 \\
\hline
\end{tabular}

\section{Interaction of RBD With ACE2 Receptor}

Earlier, the crystal structure of the RBD of SARS-CoV complexed structure had been deposited in PDB (PBD 3D0J). To understand the possible interaction between the RBD of SARS-CoV-2 with the ACE2 receptor we used 3D0J coordinates as a reference model and the RBD-AEC2 complex structure was modeled for SARS$\mathrm{CoV}-2$. This complex structure was further energy minimized and validated for bond interaction to inspect the clash in the binary complex. The minimized structure reveals the interaction regions between the $\mathrm{RBD}$ and the $\mathrm{ACE} 2$ receptor. In the RBD, interacting residues are clustered into three regions (Figure 3 ). Residues (474-488) from the first region are close enough to interact with the N-terminal region (residues 21-35) of the ACE2 receptor. Additionally, in this region two cysteine residues (480 and 488) form a disulfide bond that makes a stable loop. Residues from the second region (496-506) are in close proximity to the ACE2 receptor. This arrangement enables the ACE2 receptor to interact with two different parts of the spike protein that includes residues from 41 to 43 from the N-terminal region and residues from the 351-355 of the connecting loop of $\beta$ strands. The third region of RBD (residues 449-453) is in suitable position to interact with residues from 34 to 38 of the $\mathrm{N}$ terminal region of the ACE2 receptor. Overall residues from $449-453$ to $489-499$ of the RBD form a base of the concave surface, which is surrounded by an elevated loop on both sides (Figure 3). These elevated loops consist of 8 to 9 amino acids and they are close enough to interact with the ACE2 receptor. The modeled structure reveals that region-1 and region- 2 are located on the outer surface and exposed to solvents. Region-3 is placed somewhat in the interior and occupied next to region1 and its exposure to solvents is limited. The residues of these three interacting regions were compared with the SARS-CoV RBD complexed with the ACE2 receptor (3D0J coordinates). The amino acid sequence comparison shows that there are conserved amino acids among them (Table 2).

\section{Structure Based Antigenic Epitope Prediction}

Recent studies have shown that the N-terminal region of the S1 domain of the SARS-CoV-2 is highly variable in comparison with SARS-related coronavirus. However, the SARS-CoV-2 genome 


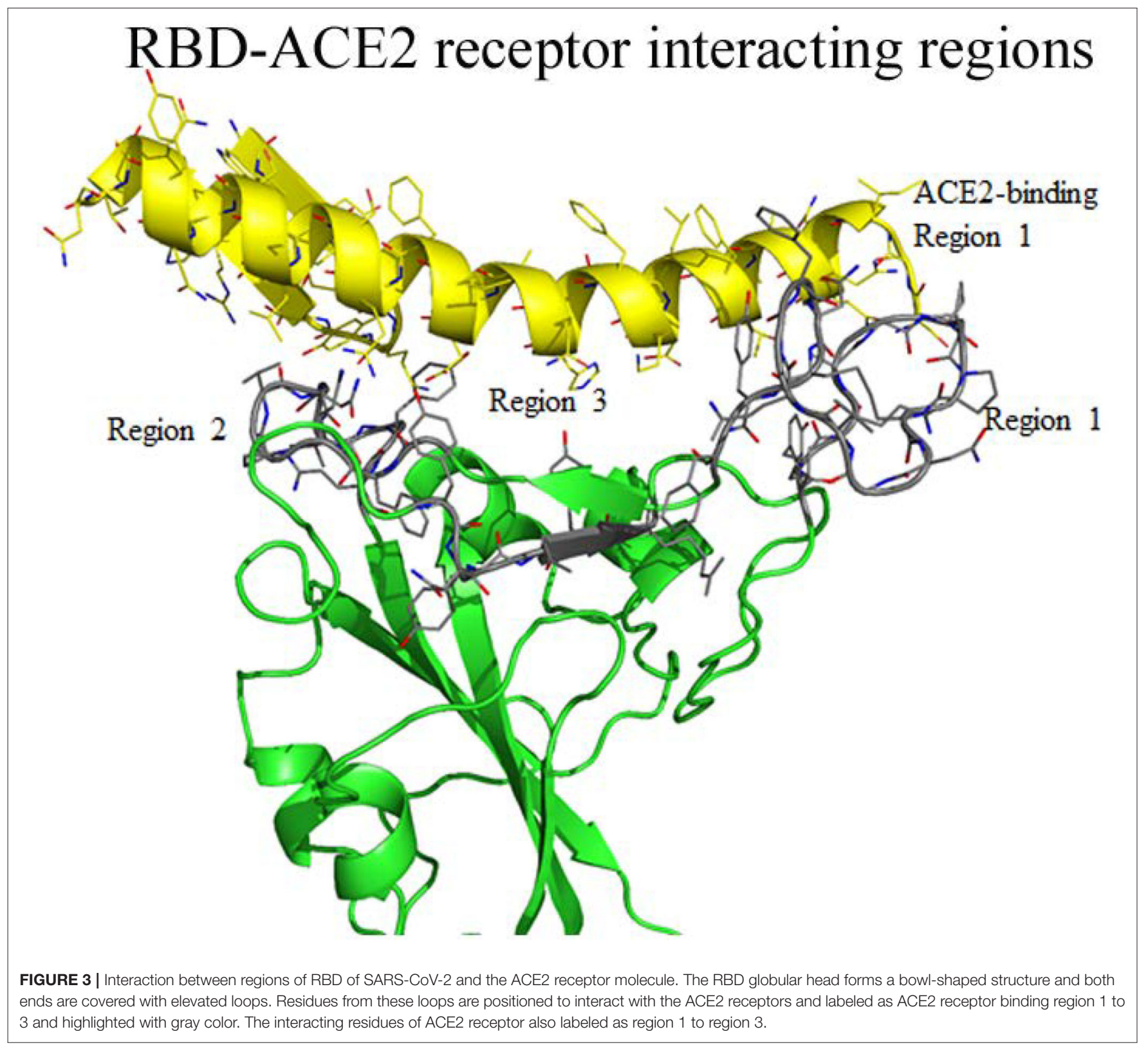

sequence reveals $79.5 \%$ sequence identity with SARS-CoV at the nucleotide level (Zhou et al., 2020), which indicates the close resemblance between them. The spike protein of the SARSCoV-2 has $76.47 \%$ of sequence identity with the spike protein of SARS-CoV (Zhou et al., 2020). The genome from different geographical origins of SARS-CoV-2 isolates have $99.9 \%$ of sequence conservation among them (Simmonds et al., 2020). Earlier, the chimeric structure of the ACE2 receptor complexed with the RBD of the SARS-CoV identified critical residues for viral infection (Li, 2008). Hua et al. (2004) identified antigenic epitopes from the spike protein of SARS-CoV and only a few peptides reacted with monoclonal antibodies raised against a fusion peptide (Hua et al., 2004). One of the antigenic epitopes was identified at residues from 446 to 461 in SARS-CoV and the equivalent region (residues 459-474) is partially conserved in SARS-CoV-2 (Table 3). Furthermore, antigenicity properties for this partially conserved region in (residues 459-474) SARSCoV-2 is unknown. Secondly, this epitope region is found to be away from the ACE-2 receptor binding region. The other predicted antigenic epitope is from the S2 domain of SARS$\mathrm{CoV}$ (residues 779-809), which is highly conserved in SARSCoV-2 as well (residues 796-826) (Table 3). In another study monoclonal antibodies were generated for the whole SARS$\mathrm{CoV}$ virus and for linear peptide antigenic epitopes; however, the monoclonal antibody generated to the whole virus cross reacted with both linear epitopes as well as with a conformational epitope (Berry et al., 2010). This study indicates that the RBD antigenic epitope is most likely exposed to the viral surface 
(Berry et al., 2010). In this study we used the spike protein structure containing a complete structure of the RBD to identify conformational epitopes.

Studies have shown that SARS-CoV-2 infected individuals have higher levels of antibodies. The secreted antibody levels are much higher in ICU patients compared to non-ICU patients (Huang et al., 2020). It is evident that cytokines probably play a major role in disease progression and severity. The increased viral replication and viral load during early stages of the infection have been correlated to an increase in apoptosis of epithelial and endothelial cells and vascular leakage, which further causes the secretion of proinflammatory cytokines through the activation of immune cells (Yang, 2020). Humoral immune response is essential for viral clearance, in particular antiviral neutralizing antibodies. Animal studies have shown that the SARS-CoV spike protein antibodies caused pulmonary injury by altering inflammatory immune responses (Liu et al., 2019). Vaccine induced pulmonary injury was also reported in Chinese rhesus monkeys, mice, and African green monkeys upon immunization of the S-protein of SARS-CoV (Bolles et al., 2011; Tseng et al., 2012; Liu et al., 2019). SARS-CoV infected individuals who developed a humoral response against the S-protein at an

TABLE 2 | Sequence comparison between modeled SARS-CoV-2 RBD-ACE2 complex and chimeric ACE2-RBD of SARS-CoV.

\begin{tabular}{lll}
\hline Region-1 & SARS-CoV (463-477) & SPDGKPCTP- PALNCY \\
& SARS-CoV-2 (474-489) & QAGSTPCNGVEGFNCY \\
& & $\ldots . . * * * \ldots * * *$ \\
Region-2 & SARS-CoV (478-495) & WPLNDYGFYTITGIGYQPYR \\
& SARS-CoV-2 (490-509) & FPLQSYGFQPTNGVGYQPYR \\
& & $* * * * * * . . * * * * * * * *$ \\
Region-3 & SARS-CoV (433-441) & TGNYNYKYR \\
& SARS-CoV-2 (446-454) & GGNYNYLYR \\
& &.$* * * * * * *$
\end{tabular}

*Indicates the conserved of residues between SARS-CoV and SARS-CoV-2, _ indicates amino acid insertion and ... indicates non-conserved residues.

Red indicates hydrophobic residues, and are either conserved or similarly charged.

Green and blue indicates hydrophilic residues, and are either conserved or similarly charged. earlier stage of infection had higher mortality compared to patients who developed a humoral response at a later stage (Zhang et al., 2006). Currently several approaches have been employed to develop antivirus immune response against the SARS-CoV-2 by various researchers and companies. These approaches include inactivated virus, viral vectors, nucleic acids, recombinant proteins, and peptide-based antigenic epitopes. Antibody dependent enhancement (ADE) of viral pathogenesis has also been reported to other viral diseases (Takada and Kawaoka, 2003; Haslwanter et al., 2017). In the case of SARSCoV-2 infection, identifying neutralizing antigenic epitopes which lack ADE is very important. Despite the promising results of the vaccine clinical trials, experts are very cautious for the complete eradication of the infection and making plea to be cautious about the cyclical illness that might stays with us, much like the flu (Corbett et al., 2020a,b; Forbes, 2020; Ho, 2020; Jackson et al., 2020).

Bioinformatic tools and a homology modeling approach have been employed here to identify an antigenic epitope from the spike protein of SARS-CoV-2. Initially, the spike protein sequence is used to predict continuous B-cell epitopes based on several criteria that include beta-turn predictions, surface accessibility, flexibility, and hydrophilic scale (Chou and Fasman, 1978; Hopp and Woods, 1983; Emini et al., 1985; Karplus and Schulz, 1985; Parker et al., 1986; Kolaskar and Tongaonkar, 1990; Lins et al., 2003; Jespersen et al., 2017) (http://tools.iedb.org/bcell). Most of the programs predictive scores are much higher in the S1 domain compared to the S2 domain. In addition, the number of predictive epitopes is also higher in the S1 domain, suggesting that more antigenic epitopes are present in the S1 domain. Furthermore, the S1 domain is more accessible to solvents or composed of a higher number of hydrophilic residues than the S2 domain. Overall these analyses indicate that the S1 domain is more accessible to be recognized by an antibody molecule or immune cells (B-cells and T-cells or other immune cells) (Figure 4). Earlier, it was shown that only a small percentage of B-cell epitopes are continuous epitopes, whereas the majority of Bcell epitopes are conformational epitopes (Van Regenmortel, 2009). To predict conformational epitopes, it is required to

TABLE 3 | Antigenic epitopes from spike protein of SARS-CoV in comparison with SARS-CoV-2 of spike protein.

\begin{tabular}{llll}
\hline Start & End & Sequence & \\
\hline 329 & 363 & FPNITNLCPFGEVFNATRFASVYAWNRKRISNCVA & No. of residues \\
369 & 393 & YNSASFSTFKCYGVSPTKLNDLCFT & 35 \\
404 & 426 & GDEVRQIAPGQTGKIADYNYKLP & 25 \\
440 & 501 & NLDSKVGGNYNYLYRLFRKSNLKPFERDISTEIYQAGSTPCNGVE GFNCYFPLQSYGFQPTN \\
516 & ELLHAPATVCGPKKSTNLVKN & 23 \\
\hline Antigenic epitope from S1 domain & SARS-CoV 446-461 & GKLRPFERDISNVPFS \\
& & SARS-CoV2 446-461 & SNLKPFERDISTEIYQ \\
Antigenic epitope from S2 domain & SARS-CoV 779-809 & GGFNFSQILPDPLKPTKRSFIEDLLFNKVT \\
& & SARS-CoV2 796-826 & GGFNFSQILPDPSKPSKRSFIEDLLFNKVT \\
\hline
\end{tabular}

The red, blue, and purple color indicates the conserved residues of hydrophobic, acidic and basic residues, respectively. The green color indicates similar charged residues. 


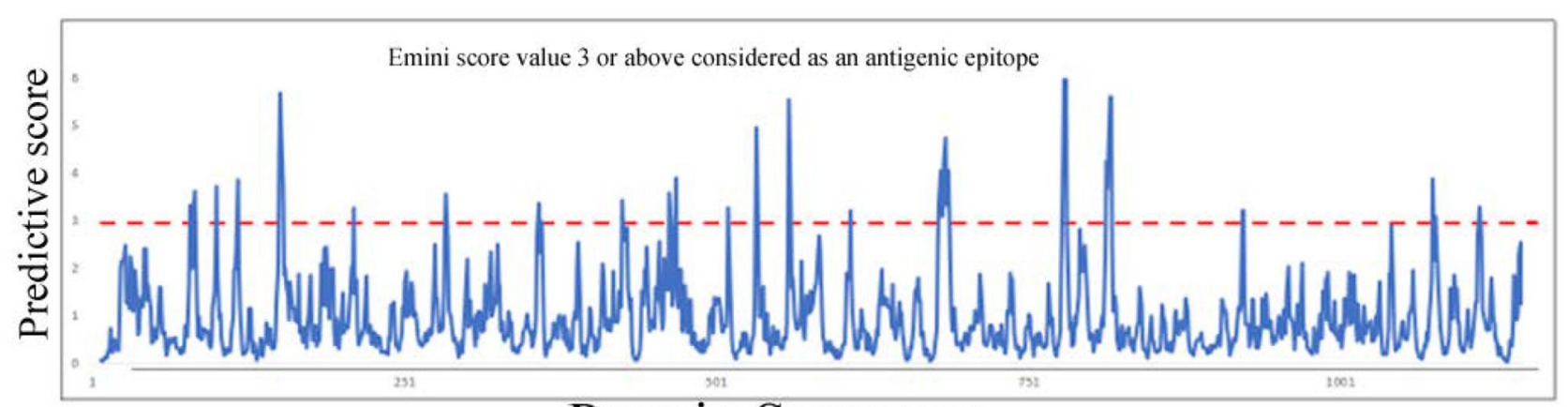

Protein Sequence

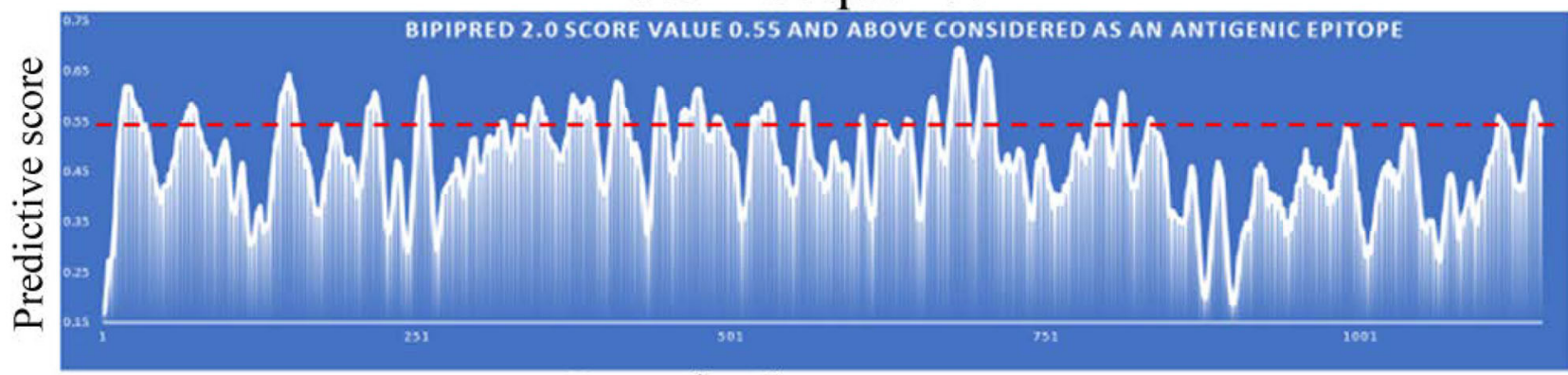

\section{Protein Sequence}

FIGURE 4 | Linear B-cell epitope prediction by various bioinformatics tools [Emini (upper panel), BepiPred-2.0 (lower panel)].

have prior knowledge about the structural information of that particular molecule.

To predict conformational epitopes from the spike protein of SARS-CoV-2 we used a current modeled structure (6 $\mathrm{vxx}$ modeled with a complete coordinate containing RBD) containing complete structural information of the RBD. In this study the model is used in two different bioinformatic tools to predict conformational antigenic epitopes based on the surface accessibility and propensity (Haste Andersen et al., 2006; Ponomarenko et al., 2008). Park et al. (2020) recently reported that SARS-CoV neutralizing antibodies are generated to conformational epitopes, whereas in MERS-CoV the neutralizing antibodies are generated to linear epitopes. The surface accessibility is an important criterion for interaction with a partner protein such as a receptor or antibody molecule. Both programs predicated three conformational antigenic epitopes in the S1 domain and 1 antigenic epitope from the S2 domain (Table 4). The predicted conformational antigenic epitopes of the RBD are compared with binding regions of the RBD-ACE2 complex of SARS-CoV (Li, 2008), as well as the predicted binding region of the RBD-ACE2 binary complex (SARS-CoV2 in this study). The predicted antigenic epitope region is consistent with the earlier reported RBD-ACE2 receptor complex of SARS-CoV (Li, 2008). We also analyzed allergenicity for both conformational and linear antigenic epitope using Allercatpro. The output results revealed that all predicted antigenic peptides lacked allergenicity property. Additionally, we have also evaluated furin cleavage site for antigenic peptides; none of the antigenic epitopes overlaps with furin cleavage site (Gioia et al., 2020).
TABLE 4 | Representative predictive antigenic epitopes.

\begin{tabular}{lllc}
\hline Start & End & Sequence & No. of residues \\
\hline 333 & 339 & TNLCPFG & 7 \\
359 & 371 & SNCVADYSVLYNS & 13 \\
376 & 385 & TFKCYGVSPT & 10 \\
430 & 435 & TGCVIA & 6 \\
488 & 495 & CYFPLQSY & 8 \\
505 & 527 & YQPYRWWLSFELLHAPATVCGP & 23 \\
\hline Start & End & Sequence & Score \\
\hline 879 & 894 & AGTITSGWTFGAGAAL & 0.97 \\
594 & 609 & GVSVITPGTNTSNQVA & 0.95 \\
257 & 272 & GWTAGAAAYYGYLQP & 0.95 \\
1,112 & 1,127 & PQIITTNTFVSGNCD & 0.95 \\
245 & 260 & HRSYLTPGDSSSGWTA & 0.92 \\
1,180 & 1,195 & QKEIDRLNEVAKNLNE & 0.92 \\
476 & 491 & GSTPCNGVEGFNCYFP & 0.91 \\
\hline 0 & & &
\end{tabular}

Orange color highlighted, and green color bolded sequences are predicted by two or three methods.

The structure based predicted antigenic epitope mostly overlaps with the predicted ACE2 receptor binding region of the RBD of SARS-CoV-2 (Figure 5). The predicted conformational epitopes are consistent with three different predicting methods. In this analysis we used both closed and open conformation of the modeled spike protein structure to predict conformational 


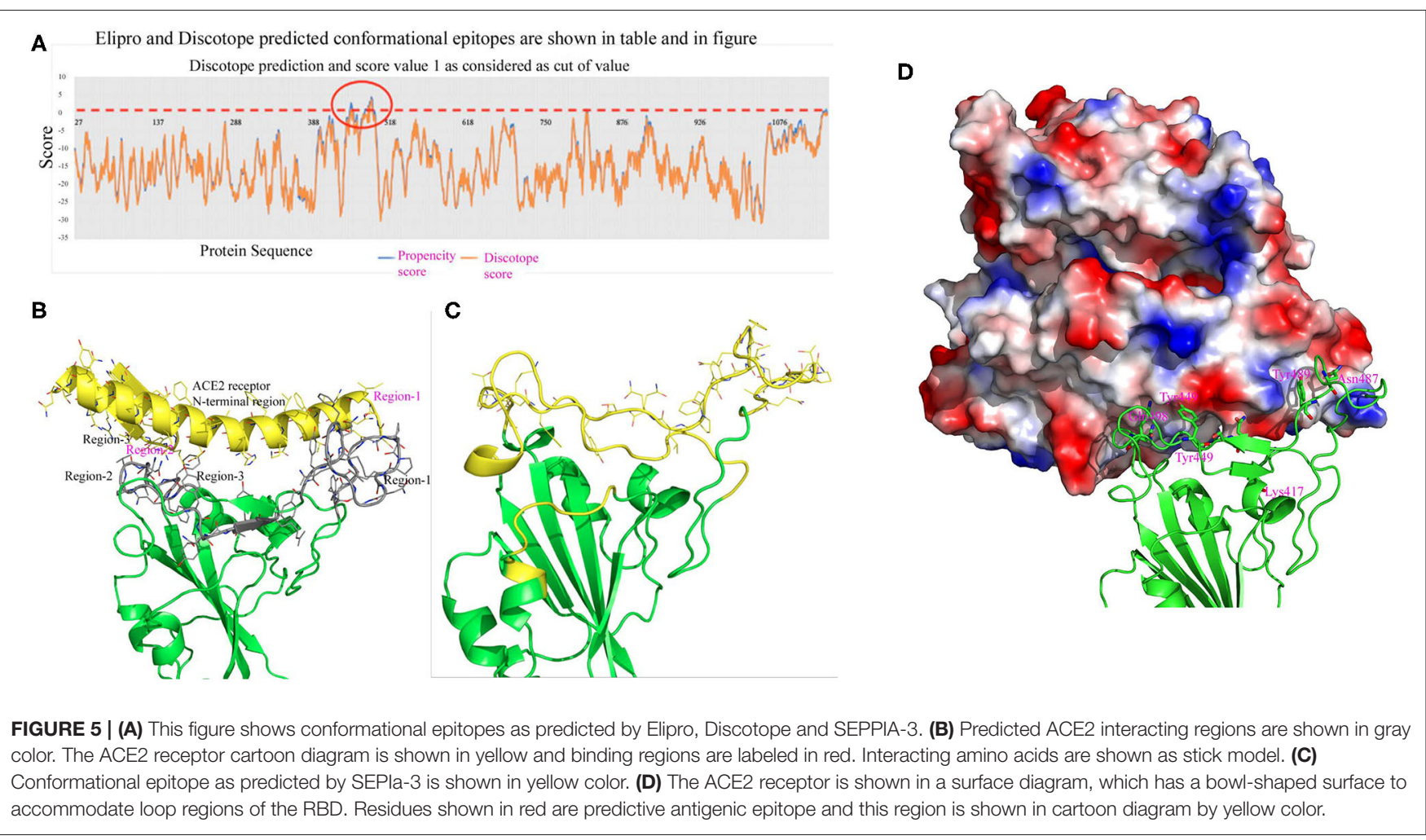

TABLE 5 | Conformational predictive scores between the open and closed conformation of the RBD of spike protein of SARS-CoV-2.

\begin{tabular}{|c|c|c|c|c|c|c|c|}
\hline No. & Chain & Start & End & Peptide & Number of residues & Score closed & Score open \\
\hline 1 & A & 469 & 490 & STEIYQAGSTPCNGVEGFNCYF & 22 & 0.838 & 0.805 \\
\hline 2 & A & 516 & 526 & ELLHAPATVCG & 11 & 0.794 & 0.786 \\
\hline 5 & $A$ & 495 & 506 & YGFQPTNGVGYQ & 12 & 0.66 & 0.66 \\
\hline 6 & $A$ & 380 & 394 & YGVSPTKLNDLCFTN & 15 & 0.642 & 0.642 \\
\hline
\end{tabular}

Sequences represented as bold are regions that interact with ACE2 receptor.

epitopes. The predictive scores for both models are very similar (Table 5). We also used 6vsb and 6vxx coordinates in structurebased antigenic epitope prediction. However, the order of prediction is changed, particularly in the RBD. The difference in the prediction is mostly due to the absence of several connecting loops in these structures, which is probably the reason for change in the predicting order. Hence, antibody production against these epitopes may be more valuable in neutralizing the viral infectivity. First, in the closed form these regions are highly solvent accessible. Secondly, in the open conformation the RBD moves away from the core region, thus there is a better chance for solvent exposure. Antibody production for these regions can possibly access the RBD both in the open as well as in the closed conformation, because these regions are solvent exposed. Furthermore, they are also involved in binding with the ACE2 receptor, which will help in decreasing the viral infectivity.

In conclusion, predicting antigenic epitopes based on structural information will help in producing neutralizing antibodies against the SARS-CoV-2 spike proteins, which will also help in designing vaccine strategy (Figure 6). Sterlin et al. (2021) provide evidence that SARS-CoV-2 mount specific IgA antibody at early stage infection, however, no data is available to demonstrate the longitudinal IgA response against SARS-CoV2 in patient recovered from infection. There is a controversy regarding the effectiveness of $\operatorname{IgA}$ and $\operatorname{IgG}$ in this infection. Recent studies have shown that IgA has more potency than IgG (Ejemel et al., 2020), however, other study does not support the potency of IgA neutralization as compared to IgG (Wang et al., 2021). IgA antibodies broadly cross react with various human coronavirus (Saad-Roy et al., 2020). Secondly, the mounting and maturation of IgA occurs at early stage of infection, but IgG mounting and maturation occurs at later stage of infection (Sterlin et al., 2021). Studies also show that SARS-Cov2 specific IgA level decreases quickly in serum in Covid-19 patients (Sterlin et al., 2021). Thus, one must be very cautious while designing neutralizing monoclonal antibodies 


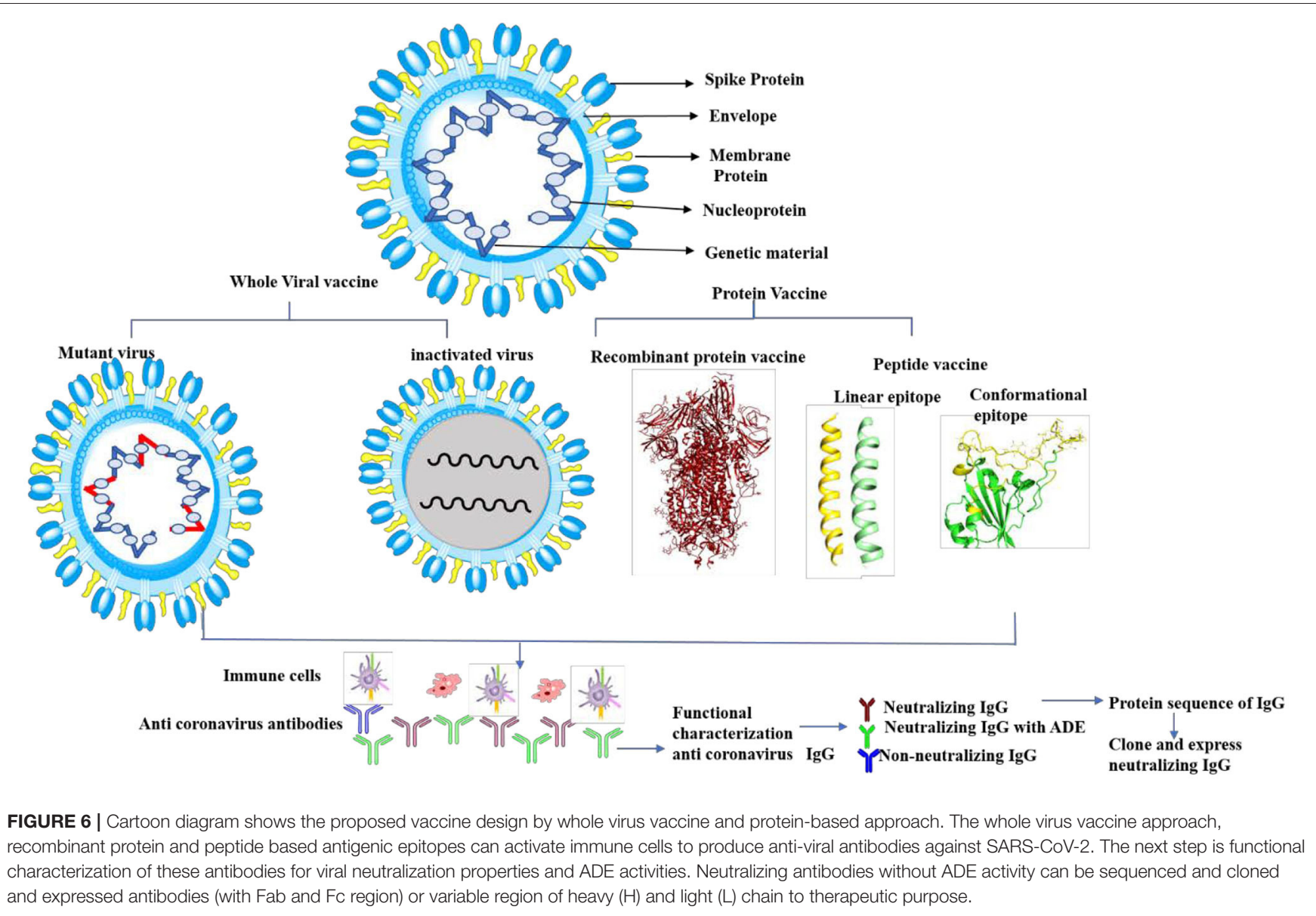

against SARS-CoV-2. A recent study shows that SARS-CoV-2 causes catastrophic microvascular injury syndrome due to the activation of the host's immune complement system (Magro et al., 2020). The immunopathological effects of antibody-dependent enhancement (ADE) have been observed in various viral infections. The same has also been reported in some COVID19 patients (Zhang et al., 2020; Zhao et al., 2020). This group of patients had severe disease, which was characterized with a higher titer of total antibodies and an enhanced IgG response. This clearly shows an ADE-mediated viral entry and the induction of severe inflammatory responses. Thus, based on our predicted epitopes, there are opportunities to design conformational based epitopes that possibly inhibit the interaction of SARS-CoV-2 to the ACE2 receptor. Furthermore, the antigenic epitopes that lack ADE viral pathogenicity are important for viral clearance. The variable region of heavy $(\mathrm{H})$ and light $(\mathrm{L})$ can be also cloned and expressed without $\mathrm{Fc}$ region for virus neutralization, which will prevent ADE viral pathogenesis (Figure 6). Experimental evidence is needed to validate this proposed hypothesis. These specific epitopes can be also used to assess antigen-specific cell stimulation or as capture antigen to detect specific antibody responses. Thus, prediction of such epitopes with bioinformatics approaches would help to distinguish infections with common cold virus vs. SARS-CoV-2.

\section{DATA AVAILABILITY STATEMENT}

The original contributions presented in the study are included in the article/supplementary material, further inquiries can be directed to the corresponding author/s.

\section{AUTHOR CONTRIBUTIONS}

SK: data analysis, writing original draft, and editing. MA: reviewing and editing manuscript. PP: sequence analysis. KG: model building, antigen epitope prediction, writing original draft, and editing the final version. All authors contributed to the article and approved the submitted version.

\section{FUNDING}

This study was funded by the U.S. FDA (E0773401).

\section{ACKNOWLEDGMENTS}

The authors would like to thank Drs. Shraddha Thakkar, Oh-Gew Kweon, and Bruce Erickson for reviewing the manuscript and providing valuable comments and suggestions. 


\section{REFERENCES}

Anthony, S. J., Gilardi, K., Menachery, V. D., Goldstein, T., Ssebide, B., Mbabazi, R., et al. (2017). Further evidence for bats as the evolutionary source of middle east respiratory syndrome coronavirus. MBio 8. doi: 10.1128/mBio.00373-17

Berry, J. D., Hay, K., Rini, J. M., Yu, M., Wang, L., Plummer, F. A., et al. (2010). Neutralizing epitopes of the SARS-CoV S-protein cluster independent of repertoire, antigen structure or mAb technology. MAbs 2, 53-66. doi: 10.4161/mabs.2.1.10788

Bolles, M., Donaldson, E., and Baric, R. (2011). SARS-CoV and emergent coronaviruses: viral determinants of interspecies transmission. Curr. Opin. Virol. 1, 624-634. doi: 10.1016/j.coviro.2011.10.012

Chen, N., Zhou, M., Dong, X., Qu, J., Gong, F., Han, Y., et al. (2020). Epidemiological and clinical characteristics of 99 cases of 2019 novel coronavirus pneumonia in Wuhan, China: a descriptive study. Lancet 395, 507-513. doi: 10.1016/S0140-6736(20)30211-7

Chou, P. Y., and Fasman, G. D. (1978). Prediction of the secondary structure of proteins from their amino acid sequence. Adv. Enzymol. Relat. Areas Mol. Biol. 47, 45-148. doi: 10.1002/9780470122921.ch2

Corbett, K. S., Edwards, D. K., Leist, S. R., Abiona, O. M., BoyogluBarnum, S., Gillespie, R. A., et al. (2020a). SARS-CoV-2 mRNA vaccine design enabled by prototype pathogen preparedness. Nature 586, 567-571. doi: 10.1038/s41586-020-2622-0

Corbett, K. S., Flynn, B., Foulds, K. E., Francica, J. R., Boyoglu-Barnum, S., Werner, A. P., et al. (2020b). Evaluation of the mRNA-1273 vaccine against SARS-CoV-2 in nonhuman Primates. N. Engl. J. Med. 383, 1544-1555. doi: 10.1056/NEJMoa2024671

Ejemel, M., Li, Q., Hou, S., Schiller, Z. A., Tree, J. A., Wallace, A., et al. (2020). A cross-reactive human IgA monoclonal antibody blocks SARS-CoV2 spike-ACE2 interaction. Nat. Commun. 11:4198. doi: 10.1038/s41467-02018058-8

Emini, E. A., Hughes, J. V., Perlow, D. S., and Boger, J. (1985). Induction of hepatitis A virus-neutralizing antibody by a virus-specific synthetic peptide. J. Virol. 55, 836-839. doi: 10.1128/JVI.55.3.836-839.1985

Forbes (2020). WHO Chief Warns Vaccine Won't End Covid-19 Pandemic As Moderna, Pfizer Announce Early Successes. Forbes. Available online at: https://www.forbes.com/sites/roberthart/2020/11/16/who-chief-warnsvaccine-wont-end-covid-19-pandemic-as-moderna-pfizer-announce- earlysuccesses/

Gioia, M., Ciaccio, C., Calligari, P., De Simone, G., Sbardella, D., Tundo, G., et al. (2020). Role of proteolytic enzymes in the COVID-19 infection and promising therapeutic approaches. Biochem. Pharmacol. 182:114225. doi: 10.1016/j.bcp.2020.114225

Gralinski, L. E., Sheahan, T. P., Morrison, T. E., Menachery, V. D., Jensen, K., Leist, S. R., et al. (2018). Complement activation contributes to severe acute respiratory syndrome coronavirus pathogenesis. MBio 9. doi: 10.1128/mBio.01753-18

Gui, M., Song, W., Zhou, H., Xu, J., Chen, S., Xiang, Y., et al. (2017). Cryoelectron microscopy structures of the SARS-CoV spike glycoprotein reveal a prerequisite conformational state for receptor binding. Cell Res. 27, 119-129. doi: $10.1038 / \mathrm{cr} .2016 .152$

Haslwanter, D., Blaas, D., Heinz, F. X., and Stiasny, K. (2017). A novel mechanism of antibody-mediated enhancement of flavivirus infection. PLoS Pathog. 13:e1006643. doi: 10.1371/journal.ppat.1006643

Haste Andersen, P., Nielsen, M., and Lund, O. (2006). Prediction of residues in discontinuous B-cell epitopes using protein 3D structures. Protein Sci. 15, 2558-2567. doi: 10.1110/ps.062405906

Ho, D. (2020). Addressing COVID-19 drug development with artificial intelligence. Adv Intell Syst. 2:2000070. doi: 10.1002/aisy.202000070

Hopp, T. P., and Woods, K. R. (1983). A computer program for predicting protein antigenic determinants. Mol. Immunol. 20, 483-489. doi: 10.1016/0161-5890(83)90029-9

Hua, R., Zhou, Y., Wang, Y., Hua, Y., and Tong, G. (2004). Identification of two antigenic epitopes on SARS-CoV spike protein. Biochem. Biophys. Res. Commun. 319, 929-935. doi: 10.1016/j.bbrc.2004.05.066

Huang, C., Wang, Y., Li, X., Ren, L., Zhao, J., Hu, Y., et al. (2020). Clinical features of patients infected with 2019 novel coronavirus in Wuhan, China. Lancet 395, 497-506. doi: 10.1016/S0140-6736(20)30183-5
Jackson, L. A., Anderson, E. J., Rouphael, N. G., Roberts, P. C., Makhene, M., Coler, R. N., et al. (2020). An mRNA vaccine against SARS-CoV-2 - preliminary report. N. Engl. J. Med. 383, 1920-1931. doi: 10.1056/NEJMoa2022483

Jespersen, M. C., Peters, B., Nielsen, M., and Marcatili, P. (2017). BepiPred2.0: improving sequence-based B-cell epitope prediction using conformational epitopes. Nucleic Acids Res. 45, W24-W29. doi: 10.1093/nar/gkx346

Karplus, P. A., and Schulz, G. E. (1985). Prediction of chain flexibility in proteins - a tool for the selection of peptide antigens. Naturwissenschafren 72, 212-213. doi: $10.1007 / \mathrm{BF} 01195768$

Kolaskar, A. S., and Tongaonkar, P. (1990). A semi-empirical method for prediction of antigenic determinants on protein antigens. FEBS Lett. 10, 172-174. doi: 10.1016/0014-5793(90)80535-Q

Li, F. (2008). Structural analysis of major species barriers between humans and palm civets for severe acute respiratory syndrome coronavirus infections. J. Virol. 82, 6984-6991. doi: 10.1128/JVI.00442-08

Lins, L., Thomas, A., and Brasseur, R. (2003). Analysis of accessible surface of residues in proteins. Protein Sci. 12, 1406-1417. doi: 10.1110/ps.0304803

Liu, L., Wei, Q., Lin, Q., Fang, J., Wang, H., Kwok, H., et al. (2019). Anti-spike IgG causes severe acute lung injury by skewing macrophage responses during acute SARS-CoV infection. JCI Insight 4:e123158. doi: 10.1172/jci.insight.123158

Magro, C., Mulvey, J. J., Berlin, D., Nuovo, G., Salvatore, S., Harp, J., et al. (2020). Complement associated microvascular injury and thrombosis in the pathogenesis of severe COVID-19 infection: a report of five cases. Transl. Res. 220, 1-13. doi: 10.1016/j.trsl.2020.04.007

Park, T., Lee, S., Kim, S., Kim, M. J., Kim, H. G., Jun, S., et al. (2020). Spike protein binding prediction with neutralizing antibodies of SARS-CoV-2. bioRXiv. doi: $10.1101 / 2020.02 .22 .951178$

Parker, J. M., Guo, D., and Hodges, R. S. (1986). New hydrophilicity scale derived from high-performance liquid chromatography peptide retention data: correlation of predicted surface residues with antigenicity and X-ray-derived accessible sites. Biochemistry 25, 5425-5432. doi: 10.1021/bi00367a013

Phan, T. (2020). Novel coronavirus: from discovery to clinical diagnostics. Infect. Genet. Evol. 79:104211. doi: 10.1016/j.meegid.2020.104211

Ponomarenko, J., Bui, H. H., Li, W., Fusseder, N., Bourne, P. E., Sette, A., et al. (2008). ElliPro: a new structure-based tool for the prediction of antibody epitopes. BMC Bioinformatics 9:514. doi: 10.1186/1471-2105-9-514

Saad-Roy, C. M., Wagner, C. E., Baker, R. E., Morris, S. E., Farrar, J., Graham, A. L., et al. (2020). Immune life history, vaccination, and the dynamics of SARS-CoV2 over the next 5 years. Science 370, 811-818. doi: 10.1126/science.abd7343

Simmonds, P., Gorbalenya, A. E., Harvala, H., Hovi, T., Knowles, N. J., Lindberg, A. M., et al. (2020). Recommendations for the nomenclature of enteroviruses and rhinoviruses. Arch. Virol. 165, 793-797. doi: 10.1007/s00705-019-04520-6

Sterlin, D., Mathian, A., Miyara, M., Mohr, A., Anna, F., Claer, L., et al. (2021). IgA dominates the early neutralizing antibody response to SARS-CoV-2. Sci. Transl. Med. 13:abd2223. doi: 10.1126/scitranslmed.abd2223

Takada, A., and Kawaoka, Y. (2003). Antibody-dependent enhancement of viral infection: molecular mechanisms and in vivo implications. Rev. Med. Virol. 13, 387-398. doi: 10.1002/rmv.405

Tortorici, M. A., and Veesler, D. (2019). Structural insights into coronavirus entry. Adv. Virus Res. 105, 93-116. doi: 10.1016/bs.aivir.2019.08.002

Tseng, C. T., Sbrana, E., Iwata-Yoshikawa, N., Newman, P. C., Garron, T., Atmar, R. L., et al. (2012). Immunization with SARS coronavirus vaccines leads to pulmonary immunopathology on challenge with the SARS virus. PLOS ONE 7:e35421. doi: 10.1371/journal.pone.0035421

Van Regenmortel, M. H. (2009). What is a B-cell epitope? Methods Mol. Biol. 524, 3-20. doi: 10.1007/978-1-59745-450-6_1

Walls, A. C., Park, Y. J., Tortorici, M. A., Wall, A., McGuire, A. T., and Veesler, D. (2020). Structure, function, and antigenicity of the SARS-CoV-2 spike glycoprotein. Cell 181, 281-292.e6. doi: 10.1016/j.cell.2020.02.058

Wang, Z., Lorenzi, J. C. C., Muecksch, F., Finkin, S., Viant, C., Gaebler, C., et al. (2021). Enhanced SARS-CoV-2 neutralization by dimeric IgA. Sci. Transl. Med. 13:eabf1555. doi: 10.1126/scitranslmed.abf1555

WHO (2015). Summary of Probable SARS Cases With Onset of Illness Form 1 November 2002 to 31 July 2003. WHO. Available online at: https://www. who.int/publications/m/item/summary-of-probable-sars-caseswith-onset- ofillness-from-1-november-2002-to-31-july-2003

WHO (2019). Middle East Respiratory Syndrome Coronavirus (MERS-CoV). WHO. Available online at: https://www.who.int/emergencies/mers-cov/en/ 
WHO (2020). Novel Coronavirus (2019- $n$ CoV). WHO. Available online at: https:// www.who.int/csr/don/05-january-2020-pneumonia-of-unkown-cause-china/ en/Google Scholar

Wrapp, D., Wang, N., Corbett, K. S., Goldsmith, J. A., Hsieh, C. L., Abiona, O., et al. (2020). Cryo-EM structure of the 2019-nCoV spike in the prefusion conformation. Science 367, 1260-1263. doi: 10.1126/science.abb2507

Xu, X., Chen, P., Wang, J., Feng, J., Zhou, H., Li, X., et al. (2020). Evolution of the novel coronavirus from the ongoing Wuhan outbreak and modeling of its spike protein for risk of human transmission. Sci. China Life Sci. 63, 457-460. doi: 10.1007/s11427-020-1637-5

Yang, M. (2020). Cell Pyroptosis, a Potential Pathogenic Mechanism of 2019-nCoV Infection. Elsevier. doi: 10.2139/ssrn.3527420

Yuan, M., Wu, N. C., Zhu, X., Lee, C. D., So, R. T. Y., Lv, H., et al. (2020). A highly conserved cryptic epitope in the receptor-binding domains of SARS-CoV-2 and SARS-CoV. Science. 368, 630-633. doi: 10.1101/2020.03.13.991570

Zhang, B., Zhou, X., Zhu, C., Song, Y., Feng, F., Qiu, Y., et al. (2020). Immune phenotyping based on the neutrophil-to-lymphocyte ratio and IgG level predicts disease severity and outcome for patients with COVID-19. Front Mol Biosci. 7:157. doi: 10.3389/fmolb.2020. 00157

Zhang, L., Zhang, F., Yu, W., He, T., Yu, J., Yi, C. E., et al. (2006). Antibody responses against SARS coronavirus are correlated with disease outcome of infected individuals. J. Med. Virol. 78, 1-8. doi: 10.1002/jmv. 20499
Zhao, J., Yuan, Q., Wang, H., Liu, W., Liao, X., Su, Y., et al. (2020). Antibody responses to SARS-CoV-2 in patients of novel coronavirus disease 2019. Clin. Infect. Dis. 71, 2027-2034. doi: 10.1101/2020.03.02.20030189

Zhou, P., Yang, X. L., Wang, X. G., Hu, B., Zhang, L., Zhang, W., et al. (2020). A pneumonia outbreak associated with a new coronavirus of probable bat origin. Nature 579, 270-273. doi: 10.1038/s41586-020-2012-7

Zhu, N., Zhang, D., Wang, W., Li, X., Yang, B., Song, J., et al. (2020). A novel coronavirus from patients with pneumonia in China, 2019. N. Engl. J. Med. 382, 727-733. doi: 10.1056/NEJMoa2001017

Disclaimer: This article reflects the views of the authors and should not be construed to represent FDA's views or policies.

Conflict of Interest: The authors declare that the research was conducted in the absence of any commercial or financial relationships that could be construed as a potential conflict of interest.

Copyright (c) 2021 Khare, Azevedo, Parajuli and Gokulan. This is an open-access article distributed under the terms of the Creative Commons Attribution License (CC $B Y)$. The use, distribution or reproduction in other forums is permitted, provided the original author(s) and the copyright owner(s) are credited and that the original publication in this journal is cited, in accordance with accepted academic practice. No use, distribution or reproduction is permitted which does not comply with these terms. 